\title{
THE PATHOLOGY AND TREATMENT OF DROWNING
}

by

Surgeon Captain S. Miles, Royal Navy

Death by drowning accounts for a yearly loss of life in this country of between 1500 and 2000 persons, mostly young people in the prime of life.

There is no doubt whatever that this significant loss of life can be greatly reduced. Study of individual incidents emphasises the fact that at least $75 \%$ of them are due to lack of elementary precautions, disregard of safety factors or gross stupidity. To reduce them, which is the fundamental aim of any water safety campaign, a three-fold attack on the problem can be made. Firstly, the circumstances which lead up to the drowning incident must be studied. Secondly, the actual process of drowning should be examined and, thirdly, there is an urgent need for first aid workers and hospital medical staffs to appreciate and apply the new techniques of resuscitation and treatment to these emergencies.

\section{WHY PEOPLE DROWN}

Man is not well adapted to the aquatic environment. He is subconsciously resistant to it. Having evolved from the sea and spending his pre-natal life in a dark fluid-filled sac, any attempt to rehabilitate himself to it is a backward step. It is against such a background of suspicion that only in relatively recent times has man showed any real interest in swimming. The adaptation even now varies greatly from one person to another. There are very many who cannot swim. There is a large majority whose prowess in the water is restricted to a length or two in the local baths or an occasional splash in the sea. These would not survive very long in adverse conditions.

Finally there are those who have come to terms with the environment, who are at ease in the water and who do not panic when in difficulties. As this numbe grows so will drowning lessen.

Furthermore, man is reluctant to realise his limitations in water and may counter his inability by reckless bravado. This may lead him into trouble and imperil the lives of his would be rescuers. It is a strange quirk in the heart of man which drives him to the help of his fellow in distress even when the chances 
of his own survival are slender.

Mechanically too man is at a disadvantage in water. The speed and endurance of the average swimmer is limited and even the expert is unable to exceed for any length of time a rate in excess of $1 \frac{1}{2}$ miles per hour and this is frequently exceeded by tide and currents.

In water, generally speaking, continuous muscular activity is needed to keep the head above water so that, depending on the swimmer and the conditions of the surface, sooner or later fatigue must result and efforts cease. The majority of men will float, but at best they remain upright with only the smallest area of the scalp out of the water, the mouth and nose remain submerged. Buoyancy depends on the salinity of the water and, generally speaking, $98 \%$ of the population are positively buoyant in sea water and $90 \%$ in fresh water. When it comes to learning to swim the minority of negatively buoyant persons have the greatest difficulty.

The Royal Life Saving Society's report of 1961 and 1962, give a good account of why men drown, but there is one very important fact which is not fully appreciated. A study of accidents on land shows that unconsciousness is a relatively common event and that less than one in a hundred of these die. In water, unless there is immediate removal, an unconscious person will drown. This is seen in practice with those who fall into water after being knocked out by a blow, or a fainting attack or who, whilst swimming, suffer some acute medical emergency, such as heart failure or epilepsy. To these must now be added a series of mishaps which may overcome the underwater swimmer, particularly the spear fisherman, who may so exert himself as to run out of oxygen underwater and lose consciousness. Even in swimming baths there have been competitions where the object has been to swim underwater as far and as fast as possible. To achieve an increase in breath holding time, competitors will hyperventilate, or overbreathe, to wash out carbon-dioxide and so delay its accumulation to the level which forces breathing to be resumed. Under these conditions, especially if there is also an emotional urge, the safe reserve of oxygen in the lungs may be exhausted before completion of the swim so that when the muscles relax on surfacing, the redistribution of blood in the circulation is such that the brain becomes further denuded of its now meagre supply of oxygen and fainting will result. Unless removed from the water the man may drown. Unlike fainting on land, where the fall itself may restore somewhat the brains blood supply, in water this postural redistribution of the circulation does not occur. It is therefore of the utmost importance that the practice of hyperventilation prior to swimming or diving under water should be condemned in the strongest possible terms. 
Drowning itself is far from being a simple process of asphyxia. Underwater it is of course impossible to breathe and this alone would have fatal results. This process is, however, in the vast majority of cases, further complicated by the actual inhalation of water into the lungs so that the type of water and any suspended matter it may contain is of great importance in determining the subsequent course of events. A full understanding, therefore, of the whole process of drowning is essential especially if, as has recently been suggested, considerations are to be made to modify the contents of water in which controlled bathing takes place. First of all, however, there are undoubtedly those cases where water does not enter the lungs and death when it ensues is due to a simple asphyxia. It is such cases of course who if rescued in time have the best chances of recovery. It is desirable, therefore, to consider drowning under three headings which may be called "Dry Drowning", "Fresh Water Drowning" and "Sea Water Drowning". There are, in addition, a very few cases where death is due not primarily to the drowning, but to the effects of sudden immersion particularly in cold water; these must receive separate consideration.

\section{Dry Drowning}

Depending largely upon the circumstances of the occasion and the condition of the victim, no water enters the lungs. This seems to be particularly true with the healthy individual who is not desperately exhausted. The presence of water in the upper respiratory passages itself promotes a protective coughing and spasm and the presence of water in the mouth also stimulates a swallowing reflex so that basically the tendency is to swallow the water rather than inhale it. The swallowing of the water seems in some cases also to lessen the urgent desire to breathe in and it is possible for complete respiratory paralysis to occur before inspiratory gasps cause water to enter the lungs. There are many cases where, after rescue and recovery, such patients have vomited copious amounts of sea water and it is these who make an uncomplicated recovery and show no evidence of lung damage when X-rayed. Death here, when it occurs, is due to a simple asphyxia. Where there is recovery such victims recall vivid hallucinations and dreams characteristic of these near terminal events. Such dreams are usually present and, to quote some examples, include panoramic views of past life, visions of relatives and friends and vivid colours and, indeed, they have been described as more pleasant than the process of returning to life and completely free from distress. In the typical near drowning event in this group there is of course the struggling stage, but when this is over the experience becomes pleasurable and painless. The life 
saving feature of these cases is possibly the acuteness of the response to the irritating effect of water in the glottis.

Quite different is the story from men and women who have drowned with inhalation of water. Their progress towards recovery when this occurs is fraught with discomfort and danger and they do not have the pleasant dreams of the former group, but frequently describe agonizing scorching pains as the water enters the lung and a sensation of burning suffocation. The consequences of water inhalation must be considered according to its nature.

\section{SEA WATER DROWNING}

When sea water enters the lungs a situation arises wherein a hypertonic saline solution is separated by a thin permeable membrane from the blood plasma. In such a situation water will be drawn from the blood into the lungs causing a haemoconcentration. In experimentally drowned animals this indeed happens and death occurs from failure of the heart muscle due to its lack of oxygen, the blood becoming so concentrated that its free circulation is impaired.

In man, as far as experience has shown, there is indeed some concentration but this is very much less marked than would be expected. The lungs themselvee irritated by sea water, react defensively and a protein exudate enters the alveoli where, together with the water and air it meets, it produces a fine foam. This is characteristic of drowning and is often seen as a very fine froth tinged with blood escaping from the mouth and nose. Such an exudate in the lungs makes oxygenation very difficult and the condition worsens. In some cases there may be apparent recovery but death may still occur several hours later from pulmonary oedema, that is a reactionary flooding of the lungs.

\section{FRESH WATER DROWNING}

Following the same argument it would be expected that, with fresh water in the lungs, the high salt content within the blood would draw this salt-free water into the circulation producing a gross dilution. Indeed, in animals this has been shown to occur with the blood containing as much as $70 \%$ of drowning fluid and the lungs remaining dry. In such cases the red blood cells are destroyed and potassium which they contain is liberated to affect the heart muscles and to cause the ventricle to fibrillate. Death from heart failure occurs in a very short time.

There is, however, very little evidence that this occurs to any great extent in man and pathologists have invariably found that post mortem examination 
of fresh water drowning shows the presence of the fine fluid which is so characteristic of the sea water drowning.

In a recent study of approximately 3,000 drowning reports, Captain Roger Fuller of the U.S. Naval Medical Corps, found no evidence whatever of ventricular fibrillation occurring in man and though in his fresh water cases there was frequently evidence that the blood was diluted, this was by no means constant and certainly never gross.

The discrepancy between the results of animal experiments and the findings in man may be due to the presence in the human lung of a substance which controls surface tension within the alveoli enabling the pulmonary blood to flow freely amongst them. This substance also tends to hinder the transfer of electrolytes across the membrane and it would seem that its dilution would neutralise this surface tension so allowing the alveoli to expand and compress the local capillary network so that the area around them would become exsanguinated making interchange between the alveoli and the blood stream unlikely. Thus claims that gross haemodilution might occur in fresh water drowning and haemoconcentration in sea water drowning, would seem to be unfounded in man.

\section{DROWNING IN CONTAMINATED WATER}

Even clean water in the lungs may promote reactionary changes, but very often the water is polluted with sand, debris, marine growths, chlorine and occasionally oil. An inflammatory reaction rapidly occurs showing a mottled appearance on X-ray. This is followed by pulmonary oedema and if inadequately treated, death. Once the first crisis of asphyxia has been overcome, this secondary reaction is the greatest hazard.

Delayed death from drowning has frequently occurred after apparent recovery even when there may have been no respiratory failure with the incident.

\section{SUDDEN DEATH ON IMMERSION}

From time to time incidents are reported where sudden immersion, usually in cold water, results in death from heart failure. One of the last cases reported was that of a schoolboy who died in the school swimming bath when the water temperature was in the low $50^{\circ} \mathrm{F}$. Experimental trials with man have shown that sudden immersion in water of $43^{\circ} \mathrm{F}$ will produce rises in blood pressure and disorders of heart rhythm has also been described even with water as warm as $59^{\circ} \mathrm{F}$. The effect seems to be due to the sudden impact of cold water 
on the neck and back of the head or possibly at the back of the throat if the mouth is open. Evidence is indeed scant and the incidence is fortunately not more than 1 in 50,000 dives into cold water. This would seem to suggest that swimming instruction should not be carried out in water below $60^{\circ} \mathrm{F}$.

\section{TREATMENT}

How can the number of these deaths be lessened?

The problem can be attacked from many directions and of fundamental importance is the spread of information about the hazards and an encouragement for all bathers to accept gracefully the many well tried safety precautions. These are too numerous to list here, but one especially would seem to be particularly worthy of mention, that is, the need to learn to swim.

Everyone will agree that no effort must be spared in planning to prevent the drowning incident, but nevertheless modern techniques and medical knowledge are now available which will greatly increase the chances of survival for the unfortunate victim, even when the circulation and respiration have failed. The key to success is prompt action where seconds count.

In the apparently drowned man the worst situation must be imagined in which respiratory and circulatory failure may be complicated by changes in the blood and damage to the lungs. For the rescuer and the first aid man on the spot, the most important action is the immediate commencement of exhaled air resuscitation. This can be started as soon as the casualty reaches shallow wate and continued on the shore. If the heart beat or pulse can be felt, well and good, but if after half-a-dozen inflations there is no improvement, if no pulse can be felt in the neck and if the pupils of the eyes are dilated, further action will be necessary. This can be done by closed chest cardiac massage for which the victim must be on a firm surface. Every second a vertical pressure with one hand over the other is made over the lower half of the breast-bone to squeeze the heart against the back bone and pump blood into the arteries. After every 6 or 8 compressions a pause is needed for inflation of the lungs.

Together these two techniques of exhaled air resuscitation and closed chest cardiac massage will keep both respiration and circulation going until the casualty can be admitted to hospital. It is essential for every near drowning case to be taken to hospital, even if apparently recovered. Many tragic deaths have occurred hours later through neglect of this precaution. In hospital new technique are available which again increase chances of survival. Cardiac massage may be ${ }_{N}^{N}$ continued and the exhaled air resuscitation be replaced by intermittent oxygen unde 
pressure, a wise precaution against later complications. The patient may be cooled to protect the brain from damage from oxygen lack. Intravenous injections of plasma may be indicated and, in extreme cases, the replacement of unbalanced blood by repeated tranfusions.

Drowning presents a challenge not only to the man in the street and first aid worker, but to the team of specialists in hospital.

With enthusiastic applications of water safety measures, wide training in resuscitation techniques and improved hospital treatment, the death rate from drowning could be halved. Add to this a nation wide drive to teach all children to swim, and the incidence will be reduced still further.

Although much remains to be done there is some cause for satisfaction. The death rate from drowning has remained fairly constant for many years and during this period there has been a marked increase in aquatic activities. More and more people visit the seaside, small boat sailing is increasing by leaps and bounds and in recent years a growing enthusiasm for underwater swimming has exposed more and more people to the risk of drowning. Great appreciation is therefore due to the many underwater organisations, clubs and municipal authorities for their efforts with water safety. With good will and enthusiasm still more could be achieved. 